\title{
Artículos
}

\section{El teletrabajo y los profesionales de la información}

\author{
Por Lourdes Barba
}

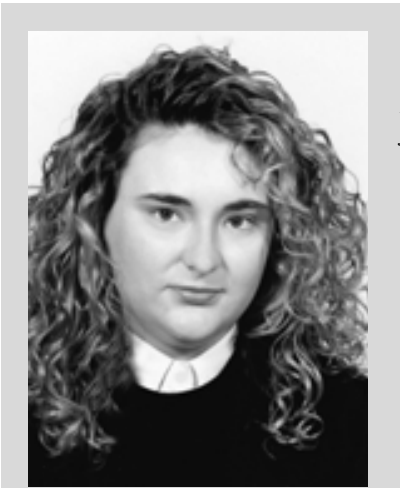

Lourdes Barba

Resumen: El teletrabajo se presentó en los 70 como una opción para la realización o desempeño de diversos puestos de trabajo que ha ido ganando adeptos a lo largo de los años hasta llegar a ser una alternativa importante en los proyectos de erradicación del desempleo tanto de la Unión Europea como de los gobiernos nacionales y que, de forma lenta pero constante, está ganando adeptos en el panorama empresarial español. El presente trabajo defiende que los profesionales de la información no pueden obviar este fenómeno social, que obtiene sus seguidores en aquellas profesiones que, como la nuestra, necesitan de las tecnologías de la información para su desarrollo, mostrando las opciones que ofrece el teletrabajo al profesional de la información, las tipologías que existen y las aplicaciones de éste al mundo documental.

Palabras clave: Teletrabajo, Profesional de la información, Informe Bangemann, Tipología del teletrabajo, Aplicaciones del teletrabajo, Unión Europea.

\section{Title: Teleworking and information professionals}

Abstract: Teleworking appeared in the 1970s as an option for certain types of work and has picked up supporters over the years. Today it is considered as an important option for reducing unemployment both at the European Union level, as well as on a national level. Although at a slower rate, it has also claimed followers from within the Spanish business sector. Information professionals cannot impede this social phenomenon that draws on professions which, like our own, rely heavily on information technologies. The present article discusses the options that teleworking can offer the information professional, the types of teleworking that currently exist, and specific applications in field of documentation.

Keywords: Teleworking, Information workers, Bangemann report, Teleworking classification, Teleworking applications, European Union.

Barba, Lourdes. "El teletrabajo y los profesionales de la información”. En: El profesional de la información, 2001, abril, v. 10, n. 4, pp. 4-13.

\section{Introducción}

El teletrabajo no es una novedad del año 2000, ni siquiera de la década de los noventa; se remonta a los años setenta y surge como solución a la "crisis del petróleo" que en ese momento sufría el mundo, y en especial EUA, en donde el economista Jack Nilles ${ }^{1}$ lo mencionó por vez primera como una opción que permitiría ahorrar el combustible del desplazamiento al lugar de trabajo. El incremento de las necesidades de autonomía y calidad de vida de los trabajadores lleva a las empresas a buscar modos de trabajo más flexibles y alternativos como el teletrabajo. La implantación de esta nueva fórmula, unida a la revolución tecnológica de finales de siglo, lo han convertido en algo mucho más que simple ahorro de combustible llegando a ser una opción de mejora socio-laboral que ofrece a aquellas personas que optan libremente por él una reducción del estrés, mayor productividad y satisfacción personal.

Llegó a ser en los noventa un fenómeno social, que debe ser analizado desde diversos puntos de vista y perspectivas. Conlleva mas innovación social que cambios en los hábitos de vida o procedimientos de trabajo, incluyéndose en una cultura de acceso y utili- zación de las tecnologías de la información (TI)². Se transformó en una opción para la realización del trabajo, que no sólo permite ahorrar combustible, sino también el tiempo de desplazamiento, el dinero de la comida fuera de casa, el del atuendo adecuado para asistir al trabajo, etc. Ofrece además una posibilidad o esperanza a colectivos con problemas para acceder a un puesto de trabajo presencial, bien sea por su minusvalía o por tener a otras personas a su cargo.

\section{«Todas las tareas que se desa- rrollan en una unidad de infor- mación del tipo que sea, pueden ser realizadas a través del tele- trabajo»}

Por otro lado, las TI se desarrollaron vertiginosamente convirtiéndose en las herramientas perfectas para la distribución de información que al mismo tiempo permiten un ahorro de tiempo y costes para la empresa, tanto en el nivel interno como externo. La informática y los ordenadores se abrían paso y se empezaba a gestar lo que hoy conocemos como sociedad de la información. Para dar una base empírica a lo expuesto 
hasta el momento, recurrimos a la tabla elaborada por el Proyecto Teldet ${ }^{3}$ (tabla 1).

La oposición llega de la mano de los sindicatos, que lo veían como una nueva forma de explotación debido a que se trabaja por objetivos y no por horas. Actualmente sin olvidar sus reticencias, lo consideran una modalidad más de trabajo que no pueden obviar por la importancia que está adquiriendo. Prueba de esto son los apartados que a ello dedican en sus páginas web5

Los gobiernos de los países más industrializados lo utilizan políticamente como sinónimo de "modernidad". Ejemplos relevantes son el "Informe Gore" de EUA o el "Informe Bangemann" de la UE. Este último nos interesa especialmente ya que es la primera mención al teletrabajo en Europa, datando del año 1994, y lo señala como la principal alternativa para paliar la "plaga de paro" que está sufriendo la UE. A partir de ese momento se han ido incluyendo en los diferentes informes y programas de la UE relativos a TI y desempleo. Actualmente tiene un papel importante en el V Programa $\operatorname{Marco}^{8}$ (1998-2003) que se vio reflejado en el informe redactado para la última Cumbre sobre paro celebrada en Lisboa en marzo de 2000 y cuyo título es "An European way for the information society". A escala nacional encontramos referencias al teletrabajo principalmente en el Plan Nacional $I+D+I^{9}$ (Investigación científica, desarrollo e innovación tecnológica) (2000-2003).

\section{Implantación en la sociedad de la información}

El sector industrial, prioritario hasta hace unos años, cede su hegemonía al de servicios. Los trabajos son menos mecánicos y repetitivos gracias al mayor uso de las TI, y se centran en la creación, transformación y envío de la información.

Como se ha expuesto anteriormente, el término sociedad de la información se popularizó en los años 90, pero como teoría surge en la década de los 60 introduciendo fuertes cambios en los conceptos que se manejaban hasta ese instante. El más relevante es que

\begin{tabular}{|l|c|c|c|c|c|}
\hline & Alemania & Francia & R. Unido & Italia & España \\
\hline $\begin{array}{l}\text { Industria y } \\
\text { construcción }\end{array}$ & 45.1 & 37.9 & 31.9 & 48.6 & $\mathbf{2 2 . 9}$ \\
\hline $\begin{array}{l}\text { Comercio y } \\
\text { distribución }\end{array}$ & 29.2 & 30.1 & 25.7 & 35.5 & $\mathbf{2 4 . 3}$ \\
\hline Banca y seguros & 38.3 & 46.6 & 48.8 & 28.9 & $\mathbf{2 3 . 8}$ \\
\hline $\begin{array}{l}\text { Administración } \\
\text { pública }\end{array}$ & 42.9 & 50.0 & 48.9 & 42.3 & $\mathbf{2 4 . 1}$ \\
\hline Otros servicios & 40.9 & 45.9 & 28.0 & 40.0 & $\mathbf{5 4 . 6}$ \\
\hline
\end{tabular}

Tabla 1. Interés en el teletrabajo por sectores industriales. Fuente: Decision makers survey, 1994 (Proyecto Teldet)
Tabla 2. Estudio de las definiciones

Campbell, J.; Froud, R. "Teleworking works!”. En: Library Association record, 1995, v. I2, pp. 654657.

Crossan, G.; Burton, P. F. "Teleworking stereotypes: a case study". En: Journal of information science, 1993, v. 19, pp. 349-362.

Davenport, E. "Groupware and improved understanding of LIS processes: some observations from experience and suggestions for future work". En: FID news bulletin, 1995, v. 45, n. 12, pp. 373-382.

Deider, C. "Teleworking and libraries". En: FID news bulletin, 1997, v. 47, n. II-12, pp. 284-288.

Mautrich, M. "Telework: also for libraries the magic world?". En: FID news bulletin, 1997, v. 47, n. II-I2, Pp. 280-283.

Scammell, A. "The information needs of teleworkers in the financial services sector". En: Managing information, 1996, v. 5, n. 3, pp. 38-40.

Stroetmann, K. A. "Telework developments and trends: a challenge for information services". En: FID news bulletin, 1997, v. 47, n. II-12, Pp. 269 274.

Teo, S. H. T.; Lim, V. K. G.; Wai, S. H. "An empirical study of attitudes towards teleworking among information technology (IT) personnel". En: International journal of information management, 1998, v. 18, n. 5, pp. 329-343.

Tung, L. L.; Turban, E. "Information technology as an enabler of telecommuting". En: International journal of information management, 1996, v. 16, n. 2, pp. 103-117.

la materia prima deja de ser algo fungible para transformarse en un concepto englobador que es la información. Surge así un cuarto sector, llamado información, que ha registrado un vertiginoso aumento hasta situarse en el puesto de cabeza en el presente. No todos los investigadores reconocen este movimiento como un nuevo sector y algunos prefieren incluirlo dentro de los servicios. Todo esto supone una evolución de los procesos de manipulación conocidos hasta el momento; la automatización ya no se define en términos de fabricación sino de "recogida de información". 


\section{Definición}

Es un mundo diferente a los círculos académicos tradicionales, por su condición de virtual desde su nacimiento, en el que se trabaja día a día y estrechamente con profesionales de otros lugares e idiomas, a los que normalmente no se conoce, pero con los que se tiene una gran afinidad investigadora; todo esto gracias obviamente a internet y a los foros de discusión o listas de correo que surcan la Red.

\section{«Las empresas han constatado que al dar mas libertad y auto- nomía al trabajador también ob- tienen de él más productividad»}

No existe una definición normalizada y universal de teletrabajo, sino que cada persona o entidad utiliza la palabra desde su propia perspectiva. La etimología $a^{10}$, tan oportuna en muchos casos, en éste necesita de ciertas connotaciones para abarcar todas las características que ofrece el término. Tras un análisis de las definiciones (ver tabla 2) podemos ofrecer una definición de síntesis de lo que es: "una modalidad de trabajo que se desarrolla a distancia del lugar convencional, utilizando para la comunicación con éste y/o con los clientes/usuarios las tecnologías de la información. Es una forma flexible a la que pueden optar los profesionales que utilicen como herramienta base para la realización de su trabajo estas tecnologías".

Si vamos a profundizar en este concepto debemos saber el enorme número de sinónimos que se pueden encontrar en la bibliografía especializada en lengua inglesa, como "telecommuting" (teledesplazamiento, es el más usado - un "commuter" es la persona que va y viene todos los días de su casa al trabajo y viceversa), "remote working" (trabajo a distancia), "net-working" (trabajo en red), "homeworking" (trabajo en casa), "flexible working" (trabajo flexible) y uno de los últimos en aparecer, "cyberworking" (cibertrabajo).

\section{Tipologías del teletrabajo}

Son diversas las clasificaciones que podemos encontrar, tanto por el tipo de contrato entre teletrabajador y contratante, como por el lugar desde el que se realiza, y ambas se combinan en cada caso para obtener el máximo beneficio para el teletrabajador.

1. Según el tipo de contrato. Dependiendo de la relación existente entre el empleado y la organización para la que realiza su labor puede ser:

- En régimen de autónomo. También conocido como por cuenta propia, son aquellos trabajadores que ofrecen sus servicios para tareas puntuales y cobran por ellos sin estar en la nómina de ninguna empresa u organización concreta. Suelen ser profesiones liberales muy relacionadas con el uso de la informática como consultores, documentalistas, contables, periodistas, diseñadores, traductores, etc.

- Por cuenta ajena. Cuando pertenece a una organización empresarial y está en nómina realizando su trabajo desde su hogar, asistiendo a la empresa tan sólo uno o dos días en semana.

2. Clasificación según el lugar de trabajo elegido. Cualquier opción elegida por el trabajador es válida siempre que le permita realizar su labor puntualmente, que puede ser desde una tumbona en la playa hasta un refugio de montaña. Existen diferentes alternativas y la bibliografía publicada al respecto diferencia básicamente tres de ellas, que son: en el propio domicilio, telecentros o centros de teletrabajo y teletrabajadores móviles o itinerantes.

Algunos autores añaden en sus listas otras, no menos válidas pero sí menos desarrolladas, que otros consideran modalidades dentro de la opción de telecentros y que son: centros de recursos compartidos, de barrio, vacacionales, oficinas satélite, etc.

— En el propio domicilio. Es el más utilizado y en el que todos pensamos al oír la palabra teletrabajo. Consiste, como su nombre indica, en realizarlo desde el hogar, con la comodidad que esto conlleva para la persona, pues puede desempeñar su labor en el horario que quiera y con el aspecto que más le apetezca.

- Telecentros. Son oficinas de recursos compartidos, que ponen a disposición del público instalaciones debidamente acondicionadas, tecnológicamente hablando, para el desarrollo de diferentes actividades. Se han convertido en una opción para el "turismo rural", y muchos ayuntamientos están invirtiendo en la instalación de estos centros ${ }^{11}$ para evitar el éxodo a las ciudades.

- Teletrabajadores móviles. Son aquellos que para la realización de su trabajo necesitan desplazarse frecuentemente para mejorar las prestaciones ofrecidas a clientes y usuarios (comerciales, personal de bibliobús, etc.). Muy frecuente en organizaciones multinacionales que ofrecen un servicio personalizado al cliente como $I B M^{12}$.

\section{Ventajas e inconvenientes del teletrabajo}

Como se ha mencionado con anterioridad, existen diversas teorías las cuales exponen sus propios listados de virtudes y problemas que, sometidos a un análisis y posterior comparativa, se unen en el siguiente resultado: 


\section{Ventajas.}

a. Para el trabajador.

- Mayor libertad: no como concepto político, sino para hacer las tareas cuando más y mejor se está dispuesto para hacerlas, aspecto que sólo conoce uno mismo y no su horario de trabajo. Las empresas han constatado que al dar más libertad y autonomía al trabajador también obtienen de él más productividad. Este aspecto se ve dimensionado en aquellos casos en que la materia prima es la información y los trabajadores que acceden a ella están mejor preparados que los profesionales de la era industrial, siendo sus aspiraciones y exigencias mayores.

- Mayor flexibilidad: unida al punto anterior hace del teletrabajador la "envidia" de sus compañeros: puede trabajar cuando quiera y donde quiera. Permite organizarse de acuerdo con sus conveniencias y la óptima realización de las tareas que le han sido encomendadas. Ya no debe elegir entre vida familiar o social y el empleo remunerado, podrá programar su jornada de forma que convivan sus obligaciones con sus devociones.

- Menor gasto: tanto para el empresario como para el teletrabajador. La reducción del tiempo invertido en desplazamientos se traduce en ahorro de dinero y de tiempo y, en la mayoría de los casos, hay que sumar los gastos de parking en zonas céntricas. El gasto en ropa es también menor, no sólo por la compra sino también por los gastos de lavandería y tintorería. Un cálculo para España nos dice que un trabajador medio puede ahorrarse en desplazamientos, ropa, comidas fuera de casa, etc., una media de 500.000 PTA al año. Ahorrar esta cantidad es mucho más fácil que obtener un incremento salarial por ese valor. Además, los ahorros no pagan impuestos.

- Opción para personas con minusvalías: los que tienen problemas físicos ven disminuidas las barreras y se equiparan al resto de trabajadores. Gracias a las TI pueden encontrar la salida laboral que siempre esperaron.

- Posibilidad de independencia: el teletrabajo bien entendido puede llevar al empleado a la imposición de sus condiciones de trabajo, para lo cual debe aprovechar muy bien las directrices que la ley de la oferta y la demanda indique en cada momento. A mayor calificación, mayor capacidad de elección. Para los mas cualificados el teletrabajo incrementa, especialmente en términos geográficos, el mercado posible para ofertar sus habilidades profesionales.

- Disponibilidad de tiempo: para desarrollar su vida familiar y social, entablar relaciones de amistad con otras personas diferentes a las del trabajo y con las que el punto de unión puede ser excepcionalmente diverso, como un hobby o un deporte. De esta forma también podrá realizar tareas impensables como trabajador.

- Vida familiar: la unidad familiar se ve apoyada ya que al menos uno de los miembros que habitualmente abandonaba el hogar para trabajar permanece en él, lo cual favorece la comunicación. En países como España, donde los jóvenes permanecen en el hogar hasta avanzada la edad laboral debido a los contratos intermitentes y mal remunerados, el teletrabajo es una forma de empleo que les ayuda (gracias al cargo de sus gastos a la economía familiar) de forma que ésta subvenciona la "oficina", los gastos de luz, agua, teléfono, equipos e incluso las telecomunicaciones. Una vez prospere el negocio, ya se podrá pensar en la opción de la independencia.

- Vida en comunidad: si el teletrabajador sólo utiliza su casa para ir a dormir y no para residir, como ocurre actualmente con la proliferación de las llamadas ciudades dormitorio, no tiene raíces y ni se siente identificado con su propio hogar. Esta falta de interés por el sistema escolar, el gobierno local y todas las cuestiones que afectan tanto al individuo como a su comunidad repercuten negativamente en el trabajo y en la decisión de permanecer en él ante otras ofertas mejor remuneradas.

\section{«Con la salida del trabajador de su entorno físico, salen do- cumentos de la organización que pueden ser confidenciales y a los cuales es más difícil proteger»}

- Reducción de los problemas de convivencia empresarial: la eliminación de un buen número de problemas laborales que pueden ser intrascendentes en apariencia (o que en ciertas culturas no tienen importancia pero en otras sí) como pueden ser las cuestiones raciales o cualesquiera que el trabajador perciba en la oficina, problemas con sus superiores y compañeros, etc.

\section{b. Para la empresa.}

- Reducción de costes: con la llegada de la revolución industrial las organizaciones han ido asumiendo cada vez más funciones, a consecuencia de las cuales han aumentado los gastos tanto fijos como de mantenimiento. Los estudios cuantitativos efectuados al respecto dicen que con el teletrabajo una empresa media española puede experimentar un ahorro en estos conceptos de millón y medio de pesetas al año por persona. De hecho la empresa ahorra porque revierte parte de los costes (espacio, energía...) en el teletrabajador. 
- Aumento de la productividad: es un parámetro de complicada medida y de aún más difícil cuantificación. Como resultado de la estimación personal de teletrabajadores y empleadores de éstos el aumento de la productividad sería del 15\%-20\%, aunque hay quienes lo elevan hasta un $30 \%$.

- Mejoras en el rendimiento y mantenimiento de los recursos humanos: la organización puede reclutar a personal que habite en zonas distantes de la oficina central sin necesidad de que haya un cambio de domicilio puesto que va a desempeñar su cargo a distancia. La empresa puede acceder a trabajadores altamente cualificados que no estén en su plantilla para labores concretas y puntuales sin preocuparse de contratarlos en su mismo espacio geográfico.

- Flexibilidad en la organización del trabajo: permite a la compañía responder mejor a los cambios que se produzcan en la demanda, pudiendo distribuir a sus empleados de la forma más adecuada obteniendo ventajas en el ámbito de actuación.

- Dirección por objetivos: el teletrabajo no se concibe sin la dirección por objetivos, por efectividad y por resultados en lugar de por presencia.

- Mejoras en la atención al usuario: los tiempos de respuesta a las solicitudes de los usuarios son menores cuando los empleados encargados de esta labor la realizan teletrabajando. Se obtiene mayor disponibilidad, mayor capacidad de reacción, de eficacia, de calidad de servicio y de imagen de centro. Las políticas flexibles agilizan la toma de decisiones y esto reduce los plazos de entrega de la información.

- Cambio de actitud frente a la contaminación: los problemas de medio ambiente han calado en la sociedad convirtiéndose en una incógnita cultural para las nuevas generaciones que no dudan en boicotear a las empresas que lo agreden. Esta sensibilización ha llevado a muchas de ellas a dar una imagen de contribución a la conservación de la naturaleza, incluyendo en sus políticas el hecho de tener teletrabajadores en nómina.

\section{c. Para la sociedad.}

- Desarrollo rural: es útil para promover el empleo en las zonas menos favorecidas o deprimidas, reduciendo así los desfases económicos y de oportunidades existentes entre la vida urbana y la rural.

- Tráfico: en los gastos de transporte hay que calcular no sólo el tiempo perdido, sino también los costes de funcionamiento del vehículo: combustible, lubricante, neumáticos, reparaciones, etc., y lo más importante: la contaminación atmosférica. Todo ello sin tener en cuenta el aumento de los accidentes de tráfico ocurridos por influencia directa o indirecta de los atas- cos, la contaminación acústica o el deterioro de la calidad de vida, factores de difícil cuantificación o valoración, pero que todos sufrimos.

- El consumo total de energía que realiza un trabajador en su casa o en un telecentro es menor que el que haría acudiendo y trabajando en un despacho o sala.

- Opciones para la enseñanza: se ha producido un "boom" en el mundo de la educación surgiendo así la teleenseñanza, una opción ampliamente aceptada por todo el mundo y de gran incidencia entre la sociedad española, como un camino para actualizarse, especializarse o simplemente desarrollar un "hobby".

\section{Inconvenientes.}

a. Para el trabajador.

- Aislamiento: esta sensación es diferente para cada individuo. Dependiendo de su espíritu y la falta de interacción diaria con sus compañeros le afectara en mayor o menor medida. La solución es sencilla, la fórmula ha de ser mixta: el trabajador deberá acudir uno o dos días por semana al centro de trabajo para contactar con sus colegas y jefes, comentar su trabajo e interrelacionarlo con ellos.

- Posible desvinculación con la empresa: al no estar presente en el día a día puede perder información de la denominada informal, de gran valor dentro de una organización: comentarios de posibles ascensos, problemas con determinados proveedores o usuarios e incluso futuras vacantes a las que podría optar.

\section{«El incremento de las necesida- des de autonomía y calidad de vida de los trabajadores lleva a las empresas a buscar modos de trabajo más flexibles y alter- nativos como el teletrabajo»}

— Inseguridad: se debe a la sensación de desprotección que puede experimentar el empleado al no existir una normativa específica y común a todos los países (algo que se está intentando consensuar desde la $C E$ ) dadas las especiales características de esta modalidad de trabajo. Debido a la importancia del tema y la necesidad de desaparición de las fronteras entre los investigadores o profesionales de cada país comunitario, para hacer frente como potencia única al resto del mundo, es preciso que sea la UE la que legisle y no cada país individualmente. Uno de los handicaps con el que se encuentran los legisladores comunitarios para algunas profesiones creativas es la protección del "copyright" de autor, algo muy complicado con los di- 
ferentes soportes en uso en la actualidad, el acceso mundial y la piratería informática.

b. Para la empresa.

- Seguridad de la información: con la salida del trabajador de su entorno físico, circulan documentos de la organización que pueden ser confidenciales y a los cuales es más difícil proteger en casa del teletrabajador. Para que exista teletrabajo debe haber una conexión entre ambos vía telemática. Este tipo de conexiones se convierte en un reto para los hackers o piratas informáticos, bien sea por el robo y posible venta de la información o por simple demostración de sus habilidades.

«El teletrabajo no cambia las funciones del bibliotecario; lo que hace es facilitar sus tareas ofreciéndole la posibilidad de realizarlas cuando y como quiera»

- Control de los empleados: surgen dificultades para la dirección y control, que varían en función del tipo de tareas a realizar y la modalidad de teletrabajo elegida. Los empresarios o jefes necesitan ver a sus empleados en el momento de "emplearse" y es muy difícil convencerlos de que si ellos no los ven, trabajan igual o mejor.

- Costes en equipamiento y comunicaciones: suponen una inversión inicial importante, concretándose con los gastos de los ordenadores y equipos necesarios para que el teletrabajador desempeñe su labor. En el caso de que acuda a los conocidos telecentros para realizarla, disminuye el fuerte gasto inicial, pero se convierte en un coste mensual, aunque menor que el de asistir al centro físico de trabajo.

En el caso de centros comunitarios de la propia empresa, como las bibliotecas de barrio dependientes de una red, por ejemplo, al precio de los equipos debemos añadir el de las líneas, cuyo importe variará según circunstancias y necesidades, que se reducen cada día debido a la liberalización de las telecomunicaciones.

- Pérdida de cohesión e identidad: puede producirse si los responsables de la organización no toman las medidas oportunas para que los teletrabajadores estén bien comunicados entre sí y con la empresa. También se deben realizar reuniones periódicas que ayuden a sentir la pertenencia e integración a la organización.

- Mayor dificultad para el trabajo en equipo: es frecuente que su introducción en una organización imponga una modificación de los sistemas de control de gestión. La flexibilidad en la ejecución del teletrabajo y su deslocalización hacen más difícil el control de las tareas y del rendimiento, tal y como se venía haciendo hasta el momento. Los equipos pueden seguir funcionando mediante el empleo de los medios telemáticos apropiados. Actualmente existen softwares que permiten llevar a cabo proyectos con participantes distribuidos geográficamente.

c. Para la sociedad.

- Decaimiento económico que se podría producir en los negocios ubicados en el centro de las ciudades al reducirse el volumen de personas que diariamente acuden al mismo, tales como restaurantes, inmobiliarias, comercios, etc.

\section{Aplicaciones al mundo documental}

Una vez presentada esta opción, se enumeran las posibilidades que ofrece a los profesionales de la información ${ }^{13}$, entendiendo por éstos especialmente a bibliotecarios y documentalistas, por ser los más relacionados con las TI y las telecomunicaciones. Para otros colectivos como museólogos y archiveros esta aplicación es más problemática debido a las especiales características de los fondos con los que trabajan, que suelen estar acogidos a condiciones de conservación y seguridad elevadas que no pueden ser reproducidas en casa del profesional. Estas posibilidades son:

- En régimen de autónomo. No pertenece a la plantilla de ninguna organización o centro y ofrece sus servicios a todo aquel dispuesto a pagar sus honorarios. Podemos convertir la posibilidad de ser teletrabajador en una nueva salida laboral para los diplomados o licenciados recién titulados en busca de su primer empleo. Con una pequeña inversión en un equipo multimedia y una conexión a internet, herramienta de la que muchos ya disponen, pueden ofrecer sus servicios como documentalistas a empresas y a otros profesionales denominados "liberales". Tanto unas como otros, aunque no se planteen contratar a un profesional a tiempo completo entre sus colaboradores, pueden necesitar servicios puntuales relacionados con la gestión de datos o información, siendo el teletrabajo la opción más fácil y barata a tener en cuenta para obtenerlos.

- Por cuenta ajena. Frente al anterior, este profesional sí tiene un jefe o encargado, pertenece a la nómina de una organización de capital público o privado y por tanto debe atenerse a unas normas y realizar su trabajo según las directrices del centro. Vivimos en una sociedad que se mueve vertiginosamente, y el profesional de la información debe estar con ella. Hasta cierto punto se puede "llegar hasta la casa" del usuario consumidor de información utilizando los medios que internet y las TI ponen a nuestro alcance, encabezados por el uso del correo electrónico.

Todas las tareas que se desarrollan en una unidad de información ${ }^{14}$ (ui) del tipo que sea, pueden ser reali- 
zadas a través del teletrabajo. Los usuarios en los que debemos pensar no son únicamente los que visitan físicamente el centro, sino todos los potenciales que pueden acceder a su página web e interesarse por los servicios que se ofrecen. Una vez que la ubicuidad deja de ser un problema, entramos de lleno en el teletrabajo. Para atender a estos usuarios no se necesita la presencia del profesional en la ui ya que puede realizar su labor desde cualquier lugar siempre que disponga de un ordenador, un módem y una conexión telefónica, que puede ser vía teléfono móvil, fijo, etc. Ya no tendrá que acudir a diario al centro; sólo una o dos veces por semana según aparezca estipulado en el contrato.

En este momento existe una gran variedad de posibilidades dependiendo del grado de implantación del teletrabajo en la ui. Si es elevado obtendremos un sistema de información dinámico, con una plantilla rotatoria que acude días alternos al centro y que periódicamente se reúne al completo para planificar ${ }^{15}$ su funcionamiento e informar y poner en común sus progresos con el resto de compañeros. A cambio el centro dispondrá de mayor espacio para los usuarios presenciales al reducirse el necesario para el personal, ahora los puestos de trabajo se compartirán por los profesionales acudiendo días distintos al lugar de trabajo. Los estu$\operatorname{dios}^{16}$ realizados al respecto garantizan un mejor comportamiento en el trato directo con el usuario puesto que el profesional habrá disminuido su irritabilidad y estrés desde que trabaja desde su hogar.

Incluso las tareas denominadas internas, tales como adquisición, catalogación o gestión del préstamo interbibliotecario se pueden realizar desde cualquier lugar con las herramientas pertinentes. El teletrabajo no cambia las funciones del bibliotecario, lo que hace es facilitárselas ofreciéndole la posibilidad de realizarlas cuando y como quiera.

1. Aplicaciones para el profesional. Si realizásemos una encuesta ${ }^{17}$ entre las personas dedicadas a estas labores obtendríamos que la mayoría de ellas realizan parte de su trabajo a través de la Red con el objeto de agilizar los procesos ${ }^{18}$. Para dejar patente la facilidad de implantación de esta modalidad, se han buscado ejemplos de cada tarea mencionada en publicaciones a congresos españoles de los últimos años; de esta forma se facilita su localización y se demuestra su accesibilidad. Los más frecuentes son:

a. Consultar fuentes de información bibliográfica: para completar los datos de identificación de los documentos solicitados por los usuarios, los cuales suelen conocer muy pocos datos sobre la obra que desean consultar. De esta forma, los profesionales garantizan que la adquisición que se va a realizar sea la correcta y cubra la demanda de los solicitantes, evitando posibles errores o confusiones. Para esta labor el Ministerio de Educación y Cultura elabora diferentes bases de datos en las que, por ejemplo, se puede consultar el país de origen, la editorial, el Isbn o Issn ${ }^{19}$. También se utilizan para comprobar cómo realizan la misma labor otros profesionales ${ }^{20}$, cómo describen un documento o simplemente para desarrollar los datos no conocidos de su ficha catalográfica.

b. Consulta de catálogos en línea: para resolver dudas que puedan surgir a la hora de catalogar o clasificar las adquisiciones. Este servicio forma parte de las ofertas de colaboración de las bibliotecas nacionales de cada país como cabecera de su sistema bibliotecario. En nuestro particular modelo de autonomías, las bibliotecas públicas designadas como cabecera del sistema autonómico también pueden ser consultadas ${ }^{21}$ así como cualquier otro sistema o ui cuyo catálogo sea accesible online, independientemente del tipo de documentos $^{22}$ que conformen sus fondos. Un ejemplo usual es averiguar el país de origen de un autor, su nombre completo, etc.

c. Consultar listas de autoridades: incluidas también entre las funciones de las bibliotecas nacionales y de los grandes centros catalográficos ${ }^{23}$, que son imprescindibles para la normalización de las menciones de responsabilidad. Son de gran interés para los centros especializados que se encuentran con problemas como el de las autoras de origen anglosajón y sus cambios de apellido según sus diferentes estados civiles.

d. Consulta de tesauros ${ }^{24}$ y listas de encabezamientos $^{25}$ : para reflejar la materia de los documentos mediante descriptores alfabéticos ${ }^{26}$ de uso común con otros centros. El empleo de esta herramienta, poco extendida entre los usuarios, facilita el diseño de la ecuación de búsqueda, reduciendo el nivel de ruido en el resultado.

e. Descargar registros bibliográficos: para no duplicar la siempre tediosa tarea de la catalogación se pueden copiar de otros catálogos online y descargarlos en el nuestro. Para realizar esta labor algunas bibliotecas se unen a modo de cooperativa para compartir sus registros, como por ejemplo las bibliotecas públicas del estado, que recurren a la Biblioteca Nacional y a Rebeca ${ }^{27}$.

f. Elaboración de bases de datos: facilitan la recuperación de información ${ }^{28}$ y permiten tanto a usuarios como a profesionales interrogar la colección diseñando estrategias de consulta complejas en las que intervengan diferentes campos. En la mayoría de catálogos en línea ${ }^{29}$ se puede observar la restricción de campos utilizables en la búsqueda, limitándose a veces sólo a título y autor, lo que produce resultados pobres o como mínimo ralentiza la recuperación. Para fomentar estas 
acciones se deberán impartir cursos de perfeccionamiento $^{30}$ a usuarios y elaborar los registros también en formato $\mathrm{html}^{31}$ para que estén a disposición de todo el mundo y en cualquier momento, a través de la página web o portal del centro.

g. Contactos con proveedores: localizados a través de los directorios y guías ${ }^{32}$ creados para las distintas profesiones y que son accesibles gratuitamente en internet. De este modo se amplía la oferta y podemos obtener mejores precios y servicios, tanto para productos bibliográficos como cualesquiera otros que sean necesarios para el funcionamiento del sistema.

h. Préstamo interbibliotecario: agilizaremos su proceso sin tener que recurrir a intermediarios como correos y mensajeros, poniéndonos en contacto con el centro elegido a través del correo electrónico ${ }^{33}$ siendo un método más rápido, seguro y barato. Esta práctica es muy utilizada por las bibliotecas especializadas y universitarias ${ }^{34}$ debido a la carestía de sus fondos y a lo recortado de sus presupuestos.

i. Fomento del asociacionismo y actualización profesional: para estar al día de las novedades de nuestro campo a través de boletines ${ }^{35}$, congresos $^{36}$ y listas de distribución ${ }^{37}$ principalmente. Uno de los problemas con los que se enfrentan los profesionales de la documentación es el aislamiento o falta de contacto tanto con otros colectivos como con investigadores especializados en el área, lo que conlleva una ausencia de comunicación con las novedades de la profesión, aspecto que comparte con el teletrabajo, pero que se ve contrarrestado con congresos $^{38}$ y listas ${ }^{39}$ que surgen despertando el interés de estos profesionales por la investigación e incluso por la aplicación de alguno de estos proyectos en su centro.

j. Evaluación de programas: consulta de $\operatorname{dudas}^{40} \mathrm{y}$ petición de consejos ${ }^{41}$ a otros profesionales, que son los que mejor nos pueden asesorar a la hora de adquirir nuevos programas o software para la gestión del sistema; obtener una demo ${ }^{42}$ del mismo a través de internet y evaluarla por parte del personal del centro; buscar a través de la Red otros centros que lo utilicen y que tengan acceso a sus aplicaciones, para navegar por ellos, pidiendo incluso a los usuarios de mayor confianza que nos den su opinión al respecto.

k. Participar en redes bibliotecarias y proyectos colectivos: para que podamos ofrecer a nuestros usuarios mejores y más completos servicios mediante la cooperación ${ }^{43}$ con otros centros. Con ello se consigue ampliar el fondo de una forma que podríamos denominar virtual al ofrecer un documento del que físicamente no disponemos, pero que en caso de necesitarlo sí podemos suministrar.
1. Colegios invisibles: la Red ofrece al profesional la posibilidad de relacionarse con otros que realicen el mismo trabajo y que estén distantes geográficamente para que pongan en común sus opiniones y aprendan los unos de los otros. De este modo se crean "colegios invisibles" que propician la investigación conjunta en vez de la individual y de los habituales estudios de casos demasiado particulares, lo que favorecería ampliamente el potencial investigador de la biblioteconomía y documentación como disciplina tecnológica. Un ejemplo importante es el proyecto Gabriel $^{44}$ que coordina todas las bibliotecas nacionales de Europa para formar una gran base de datos que recoja los textos antiguos y de difícil acceso de cada país. En España, el proyecto se denomina Memoria hispánica ${ }^{45}$.

m. Asesoría virtual: diseño, actualización y mantenimiento de páginas web; análisis, selección, redacción y estructuración del contenido de los portales o páginas que pretendan tener una base científico-documental que ofrecer a quienes los visiten; obtención, análisis, catalogación y ordenación de los archivos electrónicos de interés para los usuarios del centro.

Muchos profesionales que piensen en la posibilidad de implantación del teletrabajo en sus centros deben antes analizar los requerimientos técnicos necesarios para llevarlo a cabo y comprobar si su centro posee o puede disponer de ellos: atención a usuarios a través de internet, gestión interna y relaciones externas vía correo electrónico, catalogación compartida y elaboración de tesauros entre otras. Con esto se pretende advertir del grado de automatización requerido por un centro para llevar a cabo un proyecto de implantación de teletrabajo.

2. Aplicaciones para los usuarios. Con estos aspectos en funcionamiento, se abre un panorama de servicios ${ }^{46}$ que ofrecerles vía correo electrónico y que los convertirán en usuarios bien informados:

a. Difusión selectiva de la información: para realizar esta labor el documentalista ${ }^{47}$ deberá confeccionar un cuestionario sencillo que recoja las necesidades informativas de los usuarios de la ui, remitírselo al mayor número posible de éstos y elaborar sus perfiles documentales. Basándose en ellos realizará el envío a través de correo-e de las novedades del centro que estén relacionadas con sus temas de interés.

b. Desideratas: los usuarios solicitan los documentos que les gustaría obtener en el centro. Son opciones ${ }^{48}$ que se ponen a su servicio para estar más próximos a ellos, escuchar sus necesidades, sus ideas de mejora del servicio, de propuesta de temas de interés para bibliografías, conferencias y cualquier opción que pueda ser sometida a un estudio de implantación. Esto permitirá estrechar relaciones entre el centro y los 
usuarios que además se sentirán partícipes en su funcionamiento.

c. Catálogos en línea: este servicio ${ }^{49}$ está pensado para aquellos usuarios que, por falta de tiempo, no acuden con mayor frecuencia, perdiendo así la oportunidad de enriquecer su formación tanto profesional como personal. A éstos debemos llevarles el centro a la oficina, al comercio o a su casa ${ }^{50}$, enviándoles las novedades, la lista de actividades y todo aquello que pueda vincularle más. Así, el usuario sabrá lo que puede obtener, pudiéndolo reservar o renovar a través del correo-e.

d. Información en línea: gracias al web y al correo$\mathrm{e}$, la $\mathrm{ui}^{51}$ informa de las novedades adquiridas, las bibliografías elaboradas, las guías de lectura (normalmente sobre temas de actualidad), los webs seleccionados por su interés intrínseco y por su relación con los temas de cada usuario y del centro en general, de las actividades programadas por la biblioteca, así como de cursos sobre formación de usuarios que se pueden ofrecer en modalidad no presencial o teleformación, como ya vimos. La teleenseñanza también tiene su aplicación en este mundo. Aquellos titulados interesados en obtener los estudios de $2^{\circ}$ o $3^{\text {er }}$ ciclo, o de actualización o formación especializada, y que por el motivo que sea no pueden asistir a las clases, pueden optar por matricularse en algunas universidades que ofrecen cursos online como la Universitat Oberta de Catalunya $(U O C)^{52}$.

\section{Conclusiones}

La implantación del teletrabajo abre un abanico de posibilidades al profesional ampliando su oferta laboral y provocando un cambio en las condiciones de trabajo: tendrá el horario que decida, trabajará donde le apetezca y podrá desarrollar su labor en otros ámbitos a modo de colaboraciones para ofrecer sus conocimientos a aquellos que lo deseen. Se transformará la estructura del centro, convirtiéndose en un espacio más abierto, amigable y "familiar" para el usuario.

Llegados a este punto, nadie podrá decir que el profesional de una ui no puede desarrollar su labor en la modalidad de teletrabajo. Sólo hace falta una cuestión: proponérselo y realizar la planificación necesaria para el cambio, para lo cual se puede apoyar en la bibliografía de este trabajo.

Los profesionales deben cambiar con los tiempos y mimetizarse con la sociedad a la que sirven, que en este caso crece constantemente hacia la automatización integral; y es a través de estas tecnologías como nos debemos acercar a ellos recordándoles que siempre estaremos a su lado para cualquier necesidad informativa que les surja.

\section{Notas}

1. Nilles, J. M. "Traffic reduction by telecommuting: a status review and selected bibliography". En: Jala associates technical reports, 1988, v. 1, n. 2.

2. A partir de este momento se utilizarán las siglas TI para referirse a este concepto.

3. Consultado en: 5-04-2001.

http://europa.eu.int/search/s97.vts

4. Horner, D.; Day, P. "Labour and the information society: trades union policies for teleworking". En: Journal of information science, 1995, v. 21, n. 5, pp. 333-341.

5. Un ejemplo puede ser la página web de Ccoo. Consultado en: 5-04-2001.

http://www.ccoo.es/sindicato/mujer/ciborg.html

6. Consultado en: 5-04-2001.

http://www.ati.es/novatica/1994/jul-ago/gore110.html

7. European Commission: "Recommendations to the European Council Europe and the global information society", 1994, mayo.

8. Consultado en: 28-03-00.

http://sost.cicyt.es/programa_x.htm

9. Consultado en: 5-04-2001.

http://www.mcyt.es/sepct/plan\%5Fi\%2Bd/plani\%2Bd.htm

10. Etimológicamente, "teletrabajo" es el trabajo desde lejos. Composición del prefijo griego "tele", lejos, y del sustantivo extraído del verbo trabajar, cuyo origen viene del latín popular "tripaliare".

11. Listado de telecentros en España. Consultado en: 6-01-2001.

http://www.telecat.org/tcentros.htm

12. Consultado en: 05-04-00.

http://www.ibm.com

13. Vílchez Pardo, J. "Los bibliotecarios del siglo XXI: necesidades de formación en tecnología". En: Actas de las X jornadas bibliotecarias de Andalucía, 1998, pp. 93-100.

14. Amérigo Flores, N. "Diseño y desarrollo del Centro de documentación virtual del Parque Tecnológico de Andalucía". En: Actas de las X jornadas bibliotecarias de Andalucía, 1998, pp. 353-360.

15. Chaín Navarro, C. Gestión de información en las organizaciones. Murcia: DM: Universidad, ICE, 1998.

16. Patrón Lago, P. "Impacto social del teletrabajo: el punto de vista del teletrabajador y el teleempleador". En: Telos: revista de pensamiento sobre tecnología y sociedad, 1997, 1997, julio-septiembre, n. 50, pp. 50-61.

17. Telework. Telework Spain. Consultado en: 6-04-2001. http://www.ctv.es/USERS/gesworld/Art004.htm

18. Merlo Vega, J. A.; Sorli Rojo, Á. "Acceso a la información y suministro de documentos en la era internet". En: Actas de las X jornadas bibliotecarias de Andalucía, 1998, pp. 283-299

19. Ministerio de Educación y Cultura. Consultado en: 6-04-2001. http://www.mcu.es/bases/spa/isbn/ISBN.html

20. Alonso Castellanos, F., et al. "Nuevos modelos descriptivos de materiales cartográficos". En: Actas de las VI jornadas españolas de documentación, 1998, pp. 59-71.

21. Consultado en: 10-04-2001.

http://www.sba.junta-andalucia.es/Biblioteca_de_Andalucial biblioteca de andalucia.htm

22. Ortego de Lorenzo-Cáceres, M. del P.; Bonal Zazo, J. L. "Archivos en línea: formatos de difusión de información archivística en internet". En: Actas de las VI jornadas españolas de documentación, 1998, pp. 169-182.

23. Hoffman, S. L.; Lee, H. B.; Hatch, D. Web world of authority control. http://www.lib.byu.edu/dept/catalog/authority

24. Consultado en: 6-04-2001.

http://pci204.cindoc.csic.es/tesauros/index.html 
25. Zapico Alonso, F. "Calidad de encabezamientos en bibliotecas públicas del Estado. Sus catálogos automatizados y el usuario”. En: Actas de las X jornadas bibliotecarias de Andalucía, 1998, pp. 139-155.

26. Jiménez Rodríguez, J. E. “Consideraciones sobre indización en las bibliotecas universitarias españolas". En: Actas de las VI jornadas españolas de documentación, 1998, pp. 461-468.

27. Ministerio de Educación y Cultura. Consultado en: 6-04-2001. http://www.mcu.es/REBECA/que.html

28. Fernández Molina, J. C.; Moya Anegón, F. De. Los catálogos de acceso público en línea: el futuro de la recuperación de información bibliográfica. [Málaga]: Asociación Andaluza de Bibliotecarios, 1998.

29. Beumala, $\boldsymbol{A}$., et al. "Base de datos de recursos internet científico técnicos: Ep! (Enlaces politécnicos)". En: Actas de las VI jornadas españolas de documentación, 1998, pp. 149-168.

30. Personal del centro de documentación de Andersen Consulting en España. "El usuario de información en una empresa globalizada". En: Actas de las II jornadas andaluzas de documentación, JADoc 99, 1999, pp. 213-218.

Codina, M.; Mas, M. D.; Pallas, M. "El programa de formación de usuarios de la biblioteca de 1'Escola Tècnica Superior d'Enginyeria Industrial de Barcelona". En: Actas de las II jornadas andaluzas de documentación, JADoc 99, 1999, pp. 219-231.

31. Universidad de Cantabria. Biblioteca de la Universidad de Cantabria. Consultado en: 05-04-00.

http://www.buc.unican.es/tutoriales_cd/Guia_BD.htm

32. Consultado en: 28-03-00.

http://www.interec.com/compañial_sp.html

33. Universidad Pompeu Fabra de Barcelona. Consultado en: 05-04-00. http://www.upf.es/bib/pagines/index.htm

34. Rodríguez Gairín, J. M. "Los servicios de obtención de documentos en la universidad española: objetivos e indicadores de 1997 para una nueva metodología de trabajo". En: Actas de las VI jornadas españolas de documentación, 1998, pp. 745-754.

35. Asociación Andaluza de Bibliotecarios. Consultado en: 19-01-00. http://www.aab.es/5.htm

36. Universidad Complutense de Madrid. Consultado en: 28-03-00. http://www.aab.es/5htm>ucm.es/info/multidoc

37. Rediris. Relación de listas de distribución. Consultado en: 28-03-00. http://www.rediris.es/list/select-iris.phtml? string=documentacion

38. Recoder Sellarés, M. J.; Cid Leal, P. "Los intereses profesionales de bibliotecarios y documentalistas en España a través de su participación en las Jornadas españolas de documentación automatizada: doce años de ponencias, comunicaciones y experiencias". En: Actas de las VI jornadas españolas de documentación, 1998, pp. 219-231.

39. Merlo Vega, J. A.; Sorli Rojo, Á. "Las relaciones interprofesionales en las listas de distribución de información y documentación españolas". En: Actas de las VI jornadas españolas de documentación, 1998, pp. 597-612.

40. Fernández, E.; Fernández, I. "Canal de información para ingenieros: Ei Village”. En: Actas de las II jornadas andaluzas de documentación, JADoc 99, 1999, pp. 251-258.

41. Angós Ullate, J. M. "Necesidad de una metodología que optimice la gestión documental: estudio de un caso práctico". En: Actas de las VI jornadas españolas de documentación, 1998, pp. 109-118.

42. Como se denominan las copias de breve caducidad (suelen durar un mes) de programas comerciales para probarlos y que se pueden descargar desde los portales de sus distribuidores.

43. García Ortiz, F. M. "Redes de información educativa y pedagógica: un compromiso de progreso". En: Actas de las X jornadas bibliotecarias de Andalucía, 1998, pp. 351-361.

44. Consultado en: 04-04-00.

http://portico.bl.uk/gabriel/treasures

45. Consultado en: 04-04-00

http://www.bne.es/euro4.htm
46. Izquierdo Alonso, M.; Ruiz Abellán, J.; Piñera Lucas, J. T. "Los estudios de usuarios en los programas de gestión de calidad. Propuesta de un marco teórico integrador para el estudio del usuario de información". En: Actas de las VI jornadas españolas de documentación, 1998, pp. 433-444.

47. Martorell, B.; Seguí, R. "El servicio de difusión legislativa de la Generalitat Valenciana: la difusión selectiva de la información por internet". En: Actas de las I jornadas andaluzas de documentación, JADoc 97, 1997, pp. 189-196.

48. Gómez, R.; Prats, J. "Los servicios de investigación en la biblioteca del campus de la UPC en Terrassa (Barcelona)". En: Actas de las II jornadas andaluzas de documentación, JADoc 99, 1999, pp. 195-205.

49. Arellano, C., et al. "Análisis de estructura de sitios web: el caso de las bibliotecas universitarias andaluzas". En: Actas de las II jornadas andaluzas de documentación, JADoc 99, 1999, pp. 39-50.

50. García, C. "Evolución en el acceso a bases de datos y valores añadidos". En: Actas de las VI jornadas españolas de documentación, 1998, pp. 303-312.

51. Mano González, M. de la; Moro Cabero, M. "Los nuevos retos de la formación de usuarios: a la conquista del usuario virtual". En: Actas de las X jornadas bibliotecarias de Andalucía, 1998, pp. 635-640.

52. Universitat Oberta de Catalunya. Consultado en: 01-04-2001. http://www.uoc.es

\section{Bibliografía}

Asociación Española de Teletrabajo. Consultado en: 10-04-2000. http:www.ciberteca.es/aet

Bredin, A.; Lagatree, K. M. The home office solution: how to balance your professional and personal lives while working at home. New York: Wiley \& Sons, 1998. Isbn 0471192090.

Etips, lista de discusión en inglés. majordomo@colossus.net

European Telework Online. Consultado en: 24-01-2000.

http://www.eto.org.uk

European Community Telework. Consultado en: 24-01-2000. http://www.telework-forum.org

Federación Española de Teletrabajo. Consultado en: 10-04-2000. http://wwwfestel.org

Gordon, G. Work transformation: planning and implementing the new workplace. HNB publishing, 1998. Isbn 0966428609.

Gordon, G.; Kelly, M. Telecommuting: how to make it work for you and your company. 1998. (Fuera de publicación).

Información práctica sobre teletrabajo. Lista de discusión en español. teletrabajo-on@listas.idg.es

Iturbe, B. "El teletrabajo: un fenómeno económico y social". En: Telos: revista de pensamiento sobre tecnología y sociedad, 1997, julio-septiembre, n. 50 .

Nilles, J. M. Managing telework: strategies for managing the virtual workforce. New York: Wiley \& Sons, 1998.

Padilla Meléndez, A. Teletrabajo: dirección y organización. Madrid: Editorial Ra-ma, 1998. Isbn 84-7897-317-6.

Teletrabajo. Lista de distribución en español. teletrabajo@listserv.rediris.es

Teleworking: international perspectives: from telecommuting to the virtual organisation. Jackson, Paul J.; Ver Wielen, Jos Van (eds.). Routledge, 1998. Isbn 041517127X.

101 tips for telecommuters: successfully manage your work, team, technology and family. Dinnocenzo, D. (prol.); Fetzer, R. C. (ed.). BerrettKoheler Pub, 1999. Isbn 1576750698.

The virtual workplace. Igbaria, M.; Tan, M. (eds.). Idea Group Publishing, 1998. Isbn 1878289470. 